\title{
Über das Verhältnis der Theorie der Elementarlänge zur Quantentheorie
}

\author{
P. JoRdAN
}

Eingegangen am 17. April 1968

\begin{abstract}
This is a Discussion of the idea that a generalisation of quantum mechanics (seeming to be necessary) might require a) a new concept about possibilities of microphysical measurement; b) new mathematical structures as tools of theoretical description. Perhaps, a) could have the form that in the general case of the new theory the measurement of an observable $u$ can be performed only so far as to measure in a statistical ensemble the mean value $\bar{u}$ and the mean quadratic deviation $\overline{(\Delta u)^{2}}=\overline{u^{2}}-\bar{u}^{2}$. Perhaps, b) could be fulfilled by a new class of nonassociative, commutative algebras defined in this article. Certainly the following is only a first attempt, putting forward more questions than answers.
\end{abstract}

\section{$\S 1$}

In den letzten Jahren sind von verschiedenen Verfassern Untersuchungen zum Thema der Axiomatik der Quantenmechanik vorgelegt worden. Es sei insbesondere hingewiesen auf die Arbeiten [10, 3, 8] und die dort genannte Literatur. Es scheint mir deshalb der richtige Zeitpunkt gegeben, auf die das Thema betreffenden Überlegungen zurückzukommen, mit denen ich mich schon vor längerer Zeit zu beschäftigen begonnen habe - in entscheidenden Punkten gemeinsam mit J. v. Neumann und E. WIGNeR [4]. Die auf unsere damaligen Überlegungen erfolgte Resonanz hatte sich zunächst ganz auf die mathematische Seite der Sache beschränkt - die nichtassoziativen Algebren, deren mathematische Untersuchung ich begonnen hatte, wurden Gegenstand vieler weiterer Untersuchungen; von den amerikanischen Mathematikern sind sie als „Jordan-Algebren" bezeichnet worden. Neuerdings haben H. BRAUN und M. Köcher [2] sowie andererseits R. D. SchafER [12] dieses neue mathematische Forschungsgebiet - das später auch durch E. ARTIN wesentlich gefördert wurde - in Buchform zusammenfassend behandelt. Weitere mathematische Untersuchungen haben neuestens diesem Thema noch ganz neue Seiten abgewonnen (vgl. Kozcher [7], BraUn [1]). Anknüpfende physikalische Überlegungen sind erst seit verhältnismäßig kurzer Zeit eingeleitet worden.

Im folgenden muß ich über unsere früheren Ergebnisse kurz berichten, um die Erläuterung meiner jetzigen Beurteilung des Fragengebietes verständlich zu machen. Der Inhalt des Nachfolgenden versucht, sowohl in der begrifflichen Analyse mikrophysikalischer Verhältnisse als auch hinsichtlich der mathematischen Zusammenhänge, über das Bisherige hinaus zu gehen. Es sind nämlich durch H. Petersson [9] inzwischen 
wichtige Ergebnisse erzielt worden in der Untersuchung nichtassoziativer Algebren, welche nicht mehr zu den ,,potenzassoziativen " gehören; diese Ergebnisse sind durch S. Matsushita und den Verfasser [5] in anderer Richtung weiter verfolgt worden. Neue diesbezügliche Ergebnisse spielen in der vorliegenden Note eine Rolle.

Auf Einzelheiten der zum Thema gehörigen neuesten Arbeiten verschiedener Verfasser soll hier nicht näher eingegangen werden, obwohl die dadurch erzielten Klärungen teilweise eng mit den nachfolgend zu besprechenden Fragen verknüpft sind. Eine zusammenhängende Würdigung ginge über den Rahmen einer Abhandlung hinaus.

\section{$\S 2$}

Eine axiomatische Analyse der Quantenmechanik kann zwei verschiedene Ziele verfolgen. Einerseits ein vertieftes Verständnis dafür, warum gerade die tatsächlich vorliegende, bekannte Gestalt der Quantengesetze eine mathematisch-logisch ausgezeichnete Rolle spielt, so daß sie von gewissen sehr einfachen, allgemein gehaltenen Voraussetzungen aus als die einzige in Betracht kommende mathematische Möglichkeit erwiesen werden kann.

Zweitens kann diese Analyse darauf abzielen, etwaige Möglichkeiten einer verallgemeinernden Erweiterung der Quantentheorie zu prüfen entweder mit dem Ergebnis einer positiven Aufweisung solcher Möglichkeiten oder mit dem Ergebnis, es als unwahrscheinlich zu erkennen, daß solche Erweiterungsmöglichkeiten mathematisch existieren. So hat seinerzeit die in der Arbeit v. NEUMANN-WIGNER-JORDAN durchgeführte Analyse das mathematisch überraschende Ergebnis gehabt, daß von sehr vorsichtigen Voraussetzungen aus die durch die assoziative MatrizenMultiplikation gegebene Präzisierung der Quantenmechanik als unvermeidbare Form der Theorie zustande kommt; und J. Gunson hat kürzlich die Überzeugung ausgesprochen, daß die begriffliche und mathematische Geschlossenheit der Quantenmechanik in der Tat die mathematische Möglichkeit einer sich vom Klassischen noch weiter entfernenden Theorie zu verneinen nötige.

Für den Verfasser war seit Beginn der diesbezüglichen Untersuchungen die Vermutung leitend, daß eine künftige, außer dem Wirkungsquantum $h$ auch eine Elementarlänge voll berücksichtigende Theorie eine ähnlich tiefgreifende Veränderung des mathematischen Instrumentariums erfordern würde, wie sie beim Übergang von der klassischen zur Quantentheorie erforderlich war. Bei der Präzisierung der Quantentheorie hatten die Physiker ja das Glück, daß die Mathematiker das ganze dabei erforderlich gewordene Instrumentarium schon weitgehend ausgearbeitet hatten - die Theorie der Matrizen, der quadratischen 
Formen, der Operatoren, der Gruppen-Darstellungen, der linearen Differentialgleichungen und ihrer Eigenwerte lag schon weitgehend vor. Zwar hat die volle Ausarbeitung der Mathematik der Quanten - vor allem durch J. v. NeumanN und E. Wigner - noch erhebliche Ergänzungen hinzufügen müssen. Aber der Kerngehalt der Quanten-Mathematik war von den Mathematikern schon vorher ausführlich erforscht. Dagegen schien mir vermutbar, daß die Theorie der Elementarlänge ein noch weitgehend unbekanntes mathematisches Instrumentarium erfordern würde.

Anders ausgedrückt: Es schien mir die Existenz einer mathematischen Theorie zu vermuten, welche die uns geläufig gewordene Theorie der Matrizen- (oder Operatoren-) Algebren in ähnlicher Weise umfaßt, wie die Quantentheorie ihrerseits die klassische Theorie als Grenzfall in sich schließt. Als rein mathematische Vermutung scheint mir dies gestützt zu sein durch die im folgenden anzudeutenden Ergebnisse. Ob die damit skizzierte begriffliche und mathematische Möglichkeit tatsächlich geeignet ist, zur Förderung der Mikrophysik beizutragen, wage ich noch nicht zum Gegenstand einer Prognose zu machen. Lieber möchte ich sie als ein mathematisches Modell erwägbarer Verallgemeinerungen der Quantentheorie betrachten. Die folgenden Überlegungen enthalten demgemäß auch keinerlei Versuch, etwa zu den Problemen der Renormierung oder der Heisenbergschen Elementarteilchentheorie einen Beitrag anzubahnen.

\section{$\S 3$}

Wir denken uns ein großes Kollektiv gleichartiger mikrophysikalischer Gebilde. Es kann sich z. B. um He-Atome handeln oder um Lichtquanten oder Elektronen. Experimente, in denen Erzeugung oder Vernichtung von materiellen Teilchen vorkommt, gehören nicht in den Rahmen dieser Betrachtung, da die Zuständigkeit der eigentlichen Quantenmechanik für solche Vorgänge ungewiß ist. Das Kollektiv soll einen einheitlichen Zustand $Z$ haben - einheitlich in dem Sinne, daß eine daraus entnommene Probemenge, die dem Kollektiv gegenüber äußerst klein ist, aber doch selber bereits zahlreiche Individuen umfaßt, stets als repräsentativ für das Kollektiv im Ganzen angesehen werden darf. (Ähnliche Formulierungen hat bereits J. v. NEumanN in seinen berühmten Untersuchungen gegeben.) Während wir neben Experimenten der Hochenergie-Physik auch Experimente mit Elementumwandlung grundsätzlich ausschließen - der Einfachheit halber bleibe auch die Möglichkeit ionisierter Zustände der betrachteten Atome unberücksichtigt, obwohl sie lediglich der sprachlichen Beschreibung der gedachten Beispiele etwas vermehrte Kompliziertheit auferlegen würde -, sollen sonst für den $\mathrm{Zu}$ stand $Z$ des Kollektivs alle in Betracht kommenden Möglichkeiten zugelassen werden. Es kann sich also z. B. um ein im thermodynamischen 
Gleichgewicht mit Temperatur $T$ befindliches Kollektiv von Atomen handeln oder z. B. um den ,,reinen Fall" von Atomen, die sämtlich zur Zeit des gedachten Experimentes einem bestimmten Anregungszustand des He-Atoms entsprechen. Ebenso ist an Verhältnisse zu denken, wie sie bei der selektiven Spiegelung einer Hg-Resonanzlinie vorliegen -, wobei die beteiligten Atome sich weder im Grundzustand noch im Anregungszustand befinden, sondern statt dessen einen definierten Wert der Schwingungsphase für den entsprechenden Quantenübergang haben. Auch kann daran gedacht werden, daß der Zustand $Z$ zustande kam als Ergebnis einer Gamma-mikroskopischen Ortsbeobachtung an den Elektronen des Atoms, unter nachfolgender (für Aufnahme oder Nichtaufnahme in das Kollektiv bestimmender) Sortierung nach Ergebnissen dieses Experimentes. Obwohl diese Vorstellungen sich sehr weit vom experimentell Praktikablen entfernen, besteht kein grundsätzlicher Anlaß, die Gültigkeit der Quantenmechanik für Gedankenexperimente solcher Art zu bezweifeln.

Streng genommen wäre natürlich zu denken, daß jedes Individuum des Kollektives als Einzelnes vorliegt und einzeln zum Gegenstand eines messenden Beobachtungsvorgangs gemacht werden kann. Wenn in Wirklichkeit Messungen an einem Kollektiv gemacht werden, das als ideales Gas oder etwa als Atomstrahl vorliegt, so muß vorausgesetzt werden, daß sowohl die energetische als auch die statistische Wechselwirkung der Individuen des Kollektivs (im Sinne von Bose- oder Fermi-Statistik) vernachlässigbar ist.

Wir machen uns nun eine bestimmte Vorstellung von den Meßinstrumenten, mit denen ein Individuum des Kollektivs untersucht werden kann. Ein Meßinstrument hat zwei Seiten, welche wir als die Objektseite und die Beobachterseite bezeichnen. Die Beobachterseite können wir uns ganz grob vorstellen als einen Zeiger, der als Ergebnis der Messung jeweils auf eine bestimmte Stelle einer Skala hinweist (oder ein entsprechendes Informations-Bit auf ein Magnetband gibt). Um unsere Überlegungen einzuengen, wollen wir uns vorstellen, daß bei den betrachteten mikrophysikalischen Objekten auf jeder Meß-Skala nur endlich viele Skalenwerte erscheinen. Diese sollen durch reelle Zahlen bezeichnet werden.

Die vereinfachende Annahme nur endlich vieler „Eigenwerte“ für jede meßbare Eigenschaft unserer Objekte - oder, anders ausgedrückt, nur endlicher quantenmechanischer Matrizen - ist natürlich sehr schwerwiegend, und man kann den Verdacht vertreten, daß damit gerade entscheidend bedeutungsvolle Teile der Problematik ausgeklammert werden. Zweifellos gehört es zu den wichtigsten Tatsachen der Quantenphysik, daß die realen quantenphysikalischen Gebilde den Hilbertraum zu ihrer Beschreibung erfordern und nicht etwa nur hermitische Vektorsysteme endlicher Dimension. Wie man in einer axiomatischen Formulierung der 
Quantenmechanik von vornherein den Hilbertraum ansteuern kann, ist insbesondere von J. Gunson [3] gezeigt worden. Im folgenden sollen aber gerade diejenigen Fragen untersucht werden, die übrigbleiben, wenn man diese einschneidende Vereinfachung des Problems vornimmt.

Wenn zwei Meßinstrumente in der Beziehung stehen, daß ihre Anwendung auf eine bestimmte Art von Mikroobjekten, die in Form eines Kollektivs erläuterter Art vorliegen, immer - d. h. bei jedem der möglichen Zustände $Z$ dieses Kollektivs - statistisch gleiche Ergebnisse (an beliebiger Probemenge) ergeben, so sagen wir, daß diese beiden Meßinstrumente die gleiche meßbare Größe beobachten. Wenn wir uns nun die Aufgabe stellen, eine Algebra der an einer Objektart beobachtbaren Größen aufzustellen, so verfahren wir grundsätzlich ganz ebenso, wie etwa moderne Elektrotechniker, die für ihre Zwecke eine ,SchaltungsAlgebra" entwickeln -, wobei freilich ein Unterschied darin liegt, daß eine solche Schaltungsalgebra auf gut bekannte mathematische Gesetze hinausläuft. Es handelt sich etwa um die Matrizenalgebra von Vierpolen oder in vielen wichtigen Fällen um die Boolesche Algebra distributiver Aussagen-Verbände. (Auf den geistreichen Gedanken v. NeumanNs und BIRKHOFFs, den Übergang von der klassischen physikalischen Theorie zur Quantentheorie als eine Verallgemeinerung von distributiver Logik zu modularer Logik darzustellen, soll hier nicht eingegangen werden. Ich hoffe, darauf zurückzukommen.)

Zunächst formulieren wir ein Axiom der Theorie, und zwar unter Benutzung des Begriffs des statistischen Mittelwertes oder Erwartungswertes $\bar{u}$, welcher nicht nur von der Größe $u$ selber, sondern auch vom Zustand $Z$ des Kollektivs abhängt.

Axiom I. Wenn zwei Größen $u$ und $v$ immer, also für jeden Zustand $Z$ des fraglichen Kollektivs, übereinstimmende Erwartungswerte zeigen: $\bar{u}=\bar{v}$, so sind die beiden Größen einander gleich: $u=v$.

(Natürlich könnte man zweifeln, ob dies wirklich als ein sachhaltiges Axiom anzusehen ist oder vielmehr als eine Präzisierung der obigen Definition meßbarer Größen. Jedenfalls bedeutet aber die Anwendbarkeit einer solchen Definition einen nichttrivialen quantenphysikalischen Sachverhalt.)

Axiom II. Zu jedem Paar meßbarer Größen $u, v$ gibt es stets eine dritte $w$ (gemäß Axiom I dann eindeutig bestimmte), welche bei jedem Zustand $Z$ die Beziehung

$$
\bar{w}=\bar{u}+\bar{v}
$$

erfüllt. Wir nennen $w$ definitionsweise die Summe von $u$ und $v$ :

$$
w=u+v .
$$

Nach einem Hinweis, den ich Herrn HAAG verdanke, ist in diesem Axiom ein gewisser trivialer Anteil mitenthalten. Wenn wir Meßinstru20 Commun. math. Phys., Vol. 9 
mente $U$ und $V$ für die Größen $u$ und $v$ haben, so können wir daraus auch ein anderes Instrument machen, welches (etwa in automatisiertem Verfahren) abwechselnd $u$ und $v$ mißt. Dazu ist lediglich eine makrophysikalische - ganz zur „Beobachterseite“ gehörende - Konstruktion erforderlich, deren Möglichkeit zu erwähnen noch keinerlei Aussage über die Gesetze quantenphysikalischer Objekte bedeutet. Natürlich wird der statistische Mittelwert der so gemessenen dritten Größe dem arithmetischen Mittel der beiden einzelnen Größen entsprechen.

Es ist aber zu beachten, daß wir, wenn wir im Rahmen unserer oben festgesetzten Sprechweise bleiben wollen, das neue, dritte Instrument nur dann dem schon festgelegten Begriff eines Meßinstrumentes einordnen können, wenn die beiden Meßinstrumente $U$ und $V$ übereinstimmende Skalenwerte haben - andernfalls verlassen wir offenbar den Rahmen unserer obigen Begriffe. Das wäre natürlich durchführbar, würde aber nur eine (unzweckmäßige) Veränderung der sprachlichen Ausdrucksweise bringen, ohne am sachlichen Inhalt der Theorie etwas zu ändern. Die Aussage des Axioms II, daß man auch zwei Größen $u, v$, welche nicht übereinstimmende „Eigenwert-Spektren“ haben, im angegebenen Sinne addieren kann, ist eine tiefgreifende, inhaltvolle Aussage über die Quantenphysik als solche, die nur im Sonderfall übereinstimmender Eigenwertspektren zur bloßen Trivialität wird.

Als Motivierung dieses Axioms kann eben die bekannte Quantenmechanik mit ihren empirischen Bestätigungen angeführt werden. Wir wollen uns an die übliche, meist bevorzugte Deutung halten, daß jede (hermitische) Funktion der kanonischen Variablen eines Mikrosystems grundsätzlich eine beobachtbare Größe sei, obwohl es praktisch in der erdrückenden Mehrheit denkbarer Fälle aussichtslos wäre, die fragliche Messung wirklich zu ermöglichen. Man kann die Berechtigung dieser Deutung natürlich in Zweifel ziehen; doch dürfte dieser Zweifel schwerlich geeignet sein, einen Weg zu einer verallgemeinerten Theorie zu zeigen, welche ebenfalls mathematisch überzeugend geschlossen wäre. $(\mathrm{Da} ß$ der Spin des freien Elektrons grundsätzlich nicht im Stern-GerlachExperiment meßbar ist, bedeutet zwar eine Einschränkung des soeben Gesagten, soll aber hier nicht genauer erörtert werden.) Da Matrizen in unproblematischer Weise addiert werden können, ist bei Anerkennung der üblichen Auffassung der Quantenphysik auch die Allgemeingültigkeit des Axioms II gegeben.

Das nunmehr anzusteuernde dritte Axiom der Theorie hebt die Tatsache hervor, daß wir die Skalen-Werte eines Meßinstrumentes beliebig abändern können. Sind diese „Eigenwerte“ zunächst etwa die Zahlen $\alpha_{1}, \alpha_{2}, \ldots, \alpha_{r}$, so können wir die Skala statt dessen auch unter Benutzung einer Funktion $f(\alpha)$ ersetzen durch die abgeänderten Zahlenwerte $f\left(\alpha_{1}\right)$, $f\left(\alpha_{2}\right), \ldots, f\left(\alpha_{r}\right)$. 
Wir gebrauchen dann die Sprechweise, daß das abgeänderte Meßinstrument statt der früher gemessenen Größe $a$ die Größe $f(a)$ messe. Diese ganz innerhalb der Beobachterseite auszuführende Veränderung des Instrumentes könnte als bloße Trivialität angesehen werden, wenn sie nicht durch Axiom II eine schwerwiegende quantenphysikalische Bedeutung erhalten würde: Es wird ja nun behauptet, daß auch die Größe $f(a)$ zu jeder beliebigen anderen Größe addiert werden kann - eine Behauptung von außerordentlicher Tragweite.

Die Zulassung von Skalen-Änderungen („Umeichungen“) ergibt, daß wir aus einer beliebigen meßbaren Größe $a$ die Algebra der von ihr erzeugten Größen aufbauen können. Diese Algebra ist natürlich die ganz gewöhnliche, dem kommutativen, assoziativen und distributiven Gesetz unterliegende Alltags-Algebra. Als eine ihrer wichtigsten Eigenschaften formulieren wir:

Axiom III. Die Algebra der von einer Größe $a$ erzeugten Meßgrößen ist potenz-assoziativ:

$$
a^{\mu} a^{\nu}=a^{\mu+\nu} .
$$

Die hiermit gegebene Möglichkeit, insbesondere das Quadrat $a^{2}$ einer jeden meßbaren Größe $a$ zu bilden, benutzen wir nun, um für beliebige Größenpaare $u, v$ auch eine Multiplikation zu definieren. Die Definition lautet

$$
2 u v=(u+v)^{2}-u^{2}-v^{2} .
$$

Offenbar ist die so definierte Multiplikation kommutativ, sie darf also keineswegs mit der Matrixmultiplikation oder Operatorenmultiplikation verwechselt werden. Vielmehr gehört es gerade zur Zielsetzung der axiomatischen Analyse, aufzuklären, wieso und warum die nichtkommutative, aber assoziative Multiplikation der Matrizen (die freilich im Regelfall zweier nicht „,vertauschbarer" Matrizen gar nicht zu einer neuen meßbaren Größe, d. h. einer hermitischen Matrix führt) eine so beherrschende Rolle in der Quantentheorie spielt - der vom Bohrschen Korrespondenzprinzip aus gelungene historische Weg zum Gedanken der quantentheoretischen Matrizenrechnung soll in unserer Analyse ersetzt werden durch eine Klärung der Frage, warum die Mathematik nur diese Möglichkeit für die Formulierung der Quantengesetze zu bieten vermag, sofern die Theorie sich nicht weiter von der klassischen entfernen soll, als durch die Erfahrungstatsachen der Komplementarität erfordert wird.

Mathematisch nicht weniger wichtig als Axiom III ist

Axiom IV. Die Algebra der an einem Mikrogebilde meßbaren Größen ist formal reell; d. h. eine Summe von Quadraten kann nur dann gleich Null sein, wenn jede der quadrierten Größen selber Null ist. - Dieser berühmte Begriff der formal reellen Algebren ist von ARTIN und SCHREYER in die Mathematik eingeführt zur Kennzeichnung von Algebren, welche 
eine der wichtigsten Eigenschaften der reellen Zahlen-Algebra besitzen, ohne selber als Zahlen-Algebren definiert oder konstruiert zu sein. Die Mathematiker arbeiten in der abstrakten Algebren-Theorie gern mit dem komplizierteren Begriff der „halbeinfachen“ Algebren, der weite Verallgemeinerungsmöglichkeiten erschließt, die aber für die Physik nicht in Betracht kommen. Eine formal reelle Algebra ist auch halbeinfach; aber das Umgekehrte gilt nicht. Eine nach obigem Gedankengang definierte Algebra meßbarer Größen mu $\beta$ formal reell sein, weil wir uns ja die Skalenstriche als durch reelle Zahlen bezeichnet vorstellen.

Schließlich ist das distributive Gesetz

$$
a(b+c)=a c+a b
$$

axiomatisch einzuführen, wobei freilich seine Unentbehrlichkeit für eine mathematische Analyse der strukturellen Möglichkeiten der Quantenalgebren ein stärkeres Argument ist, als es aus anschaulich vorstellbaren Gedankenexperimenten heraus gewonnen werden könnte. Mit der Definition (2) kann man immerhin, statt (4) unmittelbar als Axiom anzunehmen, eine harmloser aussehende Forderung zugrunde legen, welche nur zwei statt drei beliebige Elemente enthält:

Axiom V. Es ist

$$
(a+b)^{2}+(a-b)^{2}=2 a^{2}+2 b^{2} .
$$

Daraus kann (4) als Folgerung abgeleitet werden.

Die Mathematiker haben vielfältig die nur einseitig distributiven Algebren untersucht, deren Prüfung auf physikalische Verwendbarkeit naheliegender Weise gelegentlich empfohlen worden ist. Da diese „Fastringe" (Bezeichnung nach Zassenhaus) jedoch an die Voraussetzung nichtkommutativer Multiplikation gebunden sind, können sie im Rahmen unserer Überlegungen keine Rolle spielen.

Das schließlich noch anzuwendende Axiom VI, welches eine endliche Basis der gesuchten Algebra vorschreibt, ist im oben besprochenen Sinne als bloßer Notbehelf anzusehen.

Die mathematische Frage, welche Strukturtypen irreduzibler Algebren bei Erfüllung aller erwähnten Axiome überhaupt möglich sind, ist seinerzeit in den Untersuchungen von J. v. NEumanN, E. Wigner und Verfasser gelöst worden. Das Ergebnis soll im folgenden ganz kurz beschrieben werden ohne Andeutung des Beweises. Es hat sich gezeigt, daß alle diese Algebren auch folgende Gesetzmäßigkeit erfüllen, die man dann zweckmäßigerweise als einfacheren Ersatz für das mathematisch schwer durchdringbare Axiom III zugrunde legen kann:

Axiom III*. Für jedes Größenpaar $a, b$ gilt die Beziehung

$$
a\left(a^{2} b\right)=a^{2}(a b) \text {. }
$$


Dies ist das definierende Axiom der sog. „Jordan-Algebren“. Man sieht leicht, daß die Potenzassoziativität (2) aus (6) als Folgerung abgeleitet werden kann. Das Umgekehrte war jedoch nur durch die erwähnte vollständige Strukturaufklärung zu erreichen, wobei das Axiom der formal reellen (oder mindestens ,halbeinfachen“) Algebra mit vorausgesetzt werden muß.

\section{$\S 4$}

In diesem Paragraphen sollen die Ergebnisse der mathematischen Analyse der obigen Axiome kurz zusammengefaßt werden. Als eine Tatsache, die uns damals ïberraschend war, zeigte sich: Obwohl die obigen Axiome scheinbar nur so schwache Aussagen machen (die allerdings durch die ihnen zugeschriebene Ausnahmslosigkeit Schärfe gewinnen), liefern sie doch nur eine sehr eingeschränkte Mannigfaltigkeit irreduzibler sie erfüllender Algebren. Bis auf einen hernach zu besprechenden Ausnahmefall sind sie durchweg konstruierbar mit Hilfe von assoziativen Algebren; nämlich in der Weise, daß man aufgrund der assoziativen, aber i. A. nicht kommutativen Multiplikation einer für die Konstruktion benutzten Hilfsalgebra eine andere, nicht mehr assoziative, aber kommutative Multiplikation definiert -, und zwar in einfachster Weise, nämlich so, da $B$ man aus zwei gegebenen hermitischen Matrizen $A, B$ nicht die beiden Produkte $A B$ und $B A$ bildet, sondern den in $A$ und $B$ symmetrischen Anteil $\frac{1}{2}(A B+B A)$. Das hat zugleich den Vorteil, daß man die Algebra grundsätzlich einschränken kann auf die hermitischen Matrizen, unter Ausschaltung der zwar als Rechenhilfe vorkommenden, aber keine physikalische Deutung erlaubenden nichthermitischen Matrizen - im Rechenvorgang der symmetrisierten, kommutativen Multiplikation verbunden, liefern zwei hermitische Matrizen stets eine wiederum hermitische.

Mit dieser mathematischen Feststellung gibt uns die durchgeführte Analyse ein Verständnis dafür, daß assoziative Algebren eine so beherrschende Rolle in der Quantentheorie spielen -, obwohl keine Möglichkeit besteht, die assoziative Multiplikation unmittelbar physikalisch, von den allgemeinen Grunderfahrungen aus als notwendig zu erkennen, so zeigt doch die Mathematik, daß diese assoziativen Algebren unentbehrliche Hilfskonstruktionen für die Verwirklichung der physikalisch geforderten Gesetze sind.

Man könnte freilich statt hermitischer Matrizen auch reell-symmetrische Matrizen nehmen - daß diese nicht ausreichen würden, der quantenphysikalischen Wirklichkeit zu entsprechen, ist in den obigen Axiomen noch nicht enthalten; wir haben es hier mit einer spezielleren, über die obige Axiomatik hinausgehenden Erfahrungstatsache zu tun. Andererseits könnte die mathematische Erfüllung obiger Axiome auch 
dadurch gegeben werden, daß man anstelle der hermitischen Matrizen solche nehmen würde, welche als Matrixelemente nicht komplexe Zahlen, sondern Quaternionen haben - mit entsprechend konjugiert-symmetrischer Anordnung der Matrixelemente. (Dies würde anscheinend die durchsichtigste Beschreibung für das freie Elektron mit Spin ergeben, unter Berücksichtigung der Unmöglichkeit, den Spin im Stern-Gerlach-Versuch zu messen.)

Der erwähnte merkwürdige Ausnahmefall einer besonderen JordanAlgebra, die (wie später durch A. A. ALBERT bewiesen wurde) nicht durch die Hilfskonstruktion über eine assoziative Algebra aufgebaut werden kann, ist mathematisch lehrreich, läßt aber keine unmittelbare physikalische Verwendung zu, obwohl auch in dieser Algebra die Begriffe von „Idempotenten“ (d. h. Größen, deren Eigenwerte nur 1 oder 0 sind) sowie entsprechende Relativwahrscheinlichkeiten ganz nach dem gewohnten Schema der Quantenmechanik definiert werden können. Zur Konstruktion dieser besonderen Algebra mußten wir statt reeller oder komplexer Zahlen bzw. Quaternionen eine seltsame Algebra heranziehen, die gewissermaßen das letzte Glied in der aufsteigenden Reihe der Verallgemeinerung reeller Zahlen zu komplexen Zahlen und dann zu Quaternionen ist: Diese aus Entdeckungen von BRIOSCHI und CAYLEY hervorgegangene, später vor allem durch Dickson, ARTIN, ZoRN untersuchte Algebra hat 8 Basiselemente. Während die komplexen Zahlen noch kommutativ sind und die Quaternionen immerhin noch assoziativ, ist diese letzte Stufe solcher Verallgemeinerungen auch nicht mehr assoziativ, sondern erfüllt statt dessen nur noch das schwächere ,,alternative" Gesetz, dessen Formulierung hier im Augenblick nicht wiederholt zu werden braucht. Es gibt jedenfalls auch bei dieser Algebra den Begriff der „konjugierten“ Elemente, und man kann deshalb Matrizen betrachten, deren Matrixelemente Cayley-Größen sind, wiederum in konjugiert-symmetrischer Anordnung. Unsere Analyse hat damals ergeben, daß derartige Matrizen von Cayley-Größen die einzige außer den oben erwähnten in Betracht kommende Möglichkeit mathematischer Erfüllung unserer Axiome liefern - aber nur dann, wenn Matrizen von nicht höherem als drittem Grade benutzt werden. (Der schwierige Beweis, daß solche Matrizen, wenn sie vom dritten Grade sind, wirklich das Axiom III erfüllen, war uns damals nicht gelungen. Er ist bald danach von A. A. Albert gegeben.)

$\mathrm{Daß}$ sich die Gültigkeit von $I I I^{*}$ (und $I I I$ ) auf diejenigen CayleyMatrizen beschränkt, welche den Grad 3 nicht überschreiten, konnte vom Verfasser später verständlich gemacht werden unter Bezugnahme auf die projektive Geometrie, die zu jeder Matrixalgebra gehört, oder ebenso zu der aus einer Matrixalgebra abgeleiteten potenzassoziativen Algebra. Die zur Algebra gehörenden „Eigenvektoren“ verhalten sich wie die Punkte 
einer projektiven Geometrie - die Geometrie hat bei einer Matrixalgebra $n$-ten Grades die Dimension $n-1$. (Im Fall unendlicher Matrizen entspricht dem natürlich der Hilbertraum.) Entsprechend gehört auch zur erwähnten Algebra von Cayley-Matrizen 3. Grades eine ebene projektive Geometrie, und das Fehlen einer entsprechenden assoziativen Algebra äußert sich in einer Verletzung des Desarguesschen Satzes in dieser ebenen Geometrie: Die Mathematikerin R. Moufang hat diese projektive ebene Geometrie tatsächlich gefunden und auf diesem Wege auch die CayleyAlgebra neu entdeckt. Bekanntlich ist aber für jede projektive Geometrie von mehr als zwei Dimensionen der Desarguessche Satz tatsächlich ein aus den sonstigen Axiomen ableitbarer Satz, und nicht - wie im ebenen Fall - ein selbständiges Axiom, das auch aufgegeben werden kann. Daher können die Cayley-Matrizen vom Grade 4 an aufwärts keine potenzassoziativen Algebren mehr sein - wie man auch durch Beispiele spezieller solcher Matrizen leicht beweisen kann.

Im Ergebnis unserer Analyse von 1934 schienen also keine neuen mathematischen Möglichkeiten für eine verallgemeinerte mikrophysikalische Theorie mehr zu bestehen. Jedoch haben neueste mathematische Arbeiten auch gewisse Algebren, welche das Axiom III nicht mehr erfüllen, einer genaueren mathematischen Untersuchung zugänglich gemacht. Ich werde darüber andeutungsweise berichten und glaube außerdem zeigen zu können, daß Algebren solcher Eigenschaften vielleicht geeignet sein könnten, eine physikalisch vernünftige Erweiterung der Theorie zu beschreiben. Trotz weitgehender Unfertigkeit der neuen, jetzt nur im ersten Ansatz vorliegenden mathematischen Theorie scheint es mir berechtigt, dies Thema zur Diskussion zu stellen.

\section{$\S 5$}

Mit der Bezeichnung

$$
[a, b, c]=(a b) c-a(b c)
$$

kann das Axiom III* offenbar auch so geschrieben werden:

$$
\left[a, b, a^{2}\right]=0 \text {. }
$$

Es ist leicht zu sehen, daß daraus auch folgender Satz gefolgert werden kann:

$$
\left[x, y^{2}, z\right]=2 y[x, y, z] \text {. }
$$

Dagegen ist es nicht möglich, umgekehrt aus (9) wieder (8) zu folgern; und der Mathematiker H. Petersson [9] hat sich an das Unternehmen gewagt, die möglichen Strukturverhältnisse derjenigen Algebren zu untersuchen, in welchen das Gesetz (9) gültig ist. Die von M. KoECHER 
vorgeschlagene Bezeichnung ,Lie-Tripel-Algebren“ für diese neue Klasse abstrakter Algebren ist von den Mathematikern allgemein angenommen worden ${ }^{1}$.

Das Ergebnis von Perersson lautet: Es gibt zwar Lie-TripelAlgebren, welche nicht auch (8) erfüllen. Aber wenn man nach ,halbeinfachen" Algebren fragt, dann ist aus (9) doch (8) - also Potenzassoziativität - zu folgern. Die schwächere Aussage, daß jede formal reelle Lie-Tripel-Algebra auch (8) erfüllt, ist von S. MAtsushita und Verfasser neu bewiesen durch Aufstellung des folgenden Satzes: Jedes Element $x$ einer Lie-Tripel-Algebra erfüllt die Beziehung

$$
\left(x^{2} x^{2}-x^{4}\right)^{2}=0 \text {. }
$$

(Wobei $x^{4}$ definiert ist gemäß $x^{n}=x x^{n-1}$ ). Obwohl somit die Lie-TripelAlgebren nicht weit hinaus führen über die potenzassoziativen, so sind sie doch lehrreich als erstes Beispiel einer der genaueren Untersuchung zugänglich gewordenen Klasse von nicht mehr potenzassoziativen Algebren - und es hat sich erwiesen, daß sie überraschend schöne und merkwürdige mathematische Eigenschaften besitzen.

Deshalb scheint es beachtenswert, daß für alle Lie-Tripel-Algebren folgende Gesetzmäßigkeit gilt, welche der Verfasser kürzlich aufgefunden hat und welche leicht zu bestätigen ist. Aus vier beliebigen Elementen einer Lie-Tripel-Algebra bilde man den Ausdruck:

$$
\left.\begin{array}{l}
L(u, v, w) \\
=[x,[u, x, v], w]+[u,[x, x, v], w]-[u, x,[v, x, w]] .
\end{array}\right\}
$$

Dann gilt stets

$$
L(u, v, w)+L(v, w, u)+L(w, u, v)=0 .
$$

Diese Gesetzmäßigkeit kann nun auch durch gewisse formal reelle (kommutative) Algebren erfüllt werden, wofür ich ein Beispiel angeben werde - dieses Beispiel ist nicht mehr potenzassoziativ, kann folglich, da es formal reell ist, auch keine Lie-Tripel-Algebra sein. Es handelt sich um ein Beispiel einer Klasse von formal-reellen Algebren, welche durch das Axiom (11), (12) gekennzeichnet ist.

Das Beispiel ist eine Algebra mit vier Basiselementen $E, F, A, S$. Darin soll $E$ die Haupteinheit sein; der Rest der Multiplikations-Tabelle ist dann:

$$
\left.\begin{array}{rl}
A^{2} & =F^{2}=F ; \quad S^{2}=2(E+A) ; \\
F A & =A ; \quad F S=A S=\frac{1}{2} S .
\end{array}\right\}
$$

Man sieht leicht, daß diese Algebra formal reell und daß z. B. $S^{2} S^{2} \neq S^{4}$ ist. Der Beweis für die Erfüllung des Axioms (11), (12) soll andernorts vorgelegt werden.

1 Die in der mathematischen Literatur ebenfalls vorkommende Bezeichnung „Lie-Tripel-Systeme“ hat jedoch verwirrender Weise eine ganz andere Bedeutung. 
Die so definierten Algebren haben Beziehungen zu den CayleyMatrizen höheren als 3. Grades, deren mathematische Theorie H. RÜHAAK [11] inzwischen zu untersuchen begonnen hat. Jedenfalls ist obiges Beispiel eine der Unteralgebren der (kommutativ definierten) Algebra aller konjugiert-symmetrischen Cayley-Matrizen 4. Grades.

\section{$\S 6$}

Ein Verzicht auf das Axiom III könnte zunächst äls physikalisch sinnwidrig angesehen werden, da uns ja niemand verbieten kann, an einer Meßskala eine willkürliche Umeichung vorzunehmen. Um hierzu einem Mißverständnis vorzubeugen, wurde schon oben betont, daß das Nichttriviale des Axioms III darin steckt, daß auch für die durch Umeichung entstehenden neuen Meßgrößen nach Axiom II unbeschränkte Addierbarkeit gefordert wird.

Es erscheint danach als eine nicht a priori zu verwerfende Möglichkeit, folgende Hypothese ins Auge zu fassen: In der zu suchenden (augenscheinlich erforderlichen) erweiterten Theorie können im allgemeinen (für die Mehrheit der beobachtbaren Größen) nicht mehr bestimmte Eigenwerte gemessen werden, die im Zuge des Beobachtungsvorgangs angenommen werden, sondern im allgemeinsten Fall beschränkt sich die Meßbarkeit einer beobachtbaren Größe $u$ auf:

1. Ermittlung des im fraglichen Zustand $Z$ des Kollektivs vorliegenden Erwartungswertes $\bar{u}$, und

2. Ermittlung des Quadrat-Mittelwertes der Streuung um diesen Erwartungswert:

$$
\overline{(\Delta u)^{2}}=\overline{u^{2}}-\bar{u}^{2} .
$$

Natürlich soll das nicht ausschließen, daß spezielle Größen auch in der erweiterten Theorie volle Meßbarkeit bewahren und in der Algebra der beobachtbaren Größen als solche Elemente auftreten, die eine eindeutige Potenzierung gestatten. Es könnte aber wohl in Betracht gezogen werden, daß für die Mehrheit aller beobachtbaren Größen nur die Möglichkeiten 1., 2. übrigbleiben, und es scheint mir verlockend, daß im Sinne unserer Überlegungen und Gedankenexperimente diese Möglichkeiten 1., 2. schon ausreichend wären, um eine Algebra der Meßgrößen aufzubauen: Wenn für jede Meßgröße $u$ auch ein Quadrat $u^{2}$ definiert ist, so sind wir ja immer noch imstande, neben der Addition auch eine Multiplikation von Meßgrößen zu definieren; es besteht dann aber keinerlei physikalischer Anlaß mehr, die entstehende Algebra für potenzassoziativ zu halten.

Es ist hervorzuheben, daß in diesem Vorstellungschema keineswegs jede Möglichkeit scharfer Messungsergebnisse zu entfallen braucht: Ein solches kann sich ergeben, indem die quadratische mittlere Abweichung 
vom Erwartungswert gleich Null ist. Zu der in der Quantenmechanik auftretenden Begrenzung für die Meßmöglichkeit von zwei verschiedenen Größen $u$ und $v$ zugleich kommt aber nun die neue Möglichkeit hinzu, daß gewisse Größen auch bei völligem Verzicht auf eine zugleich auszuführende Beobachtung einer anderen Größe die Eigenschaft haben könnten, nur mit endlicher Genauigkeit meßbar zu sein. So könnte beispielsweise die Ortsmessung an einem Elektron mit einem durch die Elementarlänge gegebenen Minimum des zugehörigen mittleren Schwankungsquadrates verknüpft sein.

In diesem Sinne scheinen mir für die Weiterentwicklung der Mikrotheorie gedankliche und mathematische Möglichkeiten vorhanden $\mathrm{zu}$ sein, deren genauere Prüfung vielleicht als empfehlenswert bezeichnet werden darf.

\section{Literatur}

1. BRAUN, H.:Doppelverhältnisse in Jordan-Algebren. (Im Erscheinen).

2. 一, u. M. KoEOHER: Jordan-Algebren. Berlin: Springer 1966.

3. Gunson, J.: Commun. math. Phys. 6, 262 (1967).

4. Jordan, P., J. v. Neumann u. E. Wigner: Ann. Math. 35, 29 (1934).

5. - u. S. Matsushita: Akad. d. Wiss. u. d. Lit. (Mainz) 1967, S. 121.

6. - Zur Theorie nichtassoziativer Algebren. (Im Erscheinen).

7. KотснеR, M.: On Lie algebras defined by Jordan algebras. Aarhus Universitet 1967.

8. Mrelnik, B.: Commun. Math. Phys. 9, 55-80 (1968).

9. Petersson, H. P.: Dissertation München 1966. Math. Z. 97, 1 (1967); 98, 104 (1967).

10. Prron, C.: Helv. Phys. Acta 37, 439 (1964).

11. RÜHAAK, H.: (In Vorbereitung).

12. Schafer, R. D.: An introduction to nonassociative algebra. New York $u$. London: Academic Press 1966.

P. JoRDAN

2000 Hamburg 13

Isestraße 123 